\title{
The Alpha Power Transformation Family: Properties and Applications
}

\author{
Mohamed E. Mead \\ Department of Statistics, Mathematics and Insurance, Faculty of Commerce, \\ Zagazig University, Egypt \\ mead9990@gmail.com \\ Gauss M. Cordeiro \\ Departamento de Estat stica Universidade Federal de Pernambuco Recife, Pernambuco, \\ Brazi \\ gauss@de.ufpe.br \\ Ahmed Z. Afify \\ Department of Statistics, Mathematics and Insurance, Benha University, Egypt \\ Ahmed.Afify@fcom.bu.edu.eg \\ Hazem Al-Mofleh \\ Department of Mathematics, Tafila Technical University, Tafila, Jordan \\ almof1hm@cmich.edu
}

\begin{abstract}
Mahdavi and Kundu (2017) introduced a family for generating univariate distributions called the alpha power transformation. They studied as a special case the properties of the alpha power transformed exponential distribution. We provide some mathematical properties of this distribution and define a four-parameter lifetime model called the alpha power exponentiated Weibull distribution. It generalizes some well-known lifetime models such as the exponentiated exponential, exponentiated Rayleigh, exponentiated Weibull and Weibull distributions. The importance of the new distribution comes from its ability to model monotone and non-monotone failure rate functions, which are quite common in reliability studies. We derive some basic properties of the proposed distribution including quantile and generating functions, moments and order statistics. The maximum likelihood method is used to estimate the model parameters. Simulation results investigate the performance of the estimates. We illustrate the importance of the proposed distribution over the McDonald Weibull, beta Weibull, modified Weibull, transmuted Weibull and exponentiated Weibull distributions by means of two real data sets.
\end{abstract}

Keywords: Alpha Power Family, Exponentiated Weibull Distribution, Maximum Likelihood, Moment, Order Statistic

\section{Introduction}

Recently, Mahdavi and Kundu (2017) proposed a new class of distributions called the alpha power transformation (APT) family. For an arbitrary parent cumulative distribution function (cdf) $G(x)$, Mahdavi and Kundu (2017) defined the cdf of the APT family (for $x \in \mathbb{R}$ ) by

$$
F_{A P T}(x)=\left\{\begin{array}{cc}
\frac{\alpha^{G(x)}-1}{\alpha-1} & \text { if } \alpha>0, \alpha \neq 1 \\
G(x) & \text { if } \quad \alpha=1 .
\end{array}\right.
$$


The probability density function (pdf) corresponding to (1) is

$$
f_{A P T}(x)=\left\{\begin{array}{ccc}
\frac{\log \alpha}{\alpha-1} \alpha^{G(x)} g(x) & \text { if } & \alpha>0, \alpha \neq 1 \\
g(x) & \text { if } & \alpha=1 .
\end{array}\right.
$$

In section 2 and 3, we denote by $X \sim \operatorname{APT}(\alpha)$ a random variable having pdf (2). Weighted distributions play an important role in statistical distribution theory. Clearly, for $\alpha \neq 1, f_{A P T}(x)$ is a weighted version of $g(x)$, where the weight function is $w(x ; \alpha)=\alpha^{G(x)}$, namely

$$
f_{A P T}(x)=\frac{1}{M} w(x ; \alpha) g(x)
$$

where the normalizing constant is $M=E[w(X ; \alpha)]$. Finally, we offer some conclusions in Section 8.

Here, $w(x ; \alpha)$ can be increasing or decreasing depending on whether $\alpha>1$ or $\alpha<1$. A simple interpretation of the APT family, for a non-negative random variable $X$, is as follows. Suppose a realization $x$ of $X$ enters the investigator's record with probability proportional to $w(x ; \alpha)$, say

$$
\frac{\operatorname{Prob}(\operatorname{Recording} \mid X=y)}{\operatorname{Prob}(\operatorname{Recording} \mid X=x)}=\frac{w(y ; \alpha)}{w(x ; \alpha)} .
$$

Here, the parameter $\alpha$ represents the recording mechanism. If $Y$ represents the random variable of the investigator's records, then $Y$ has the pdf (2). Finally, we offer some conclusions in Section 8.

Mahdavi and Kundu (2017) did not provide general mathematical properties of their family. Our aim is to obtain some structural properties of the APT family. We derive very simple linear representations for the pdf and cdf. We obtain explicit expressions for the ordinary and incomplete moments, moment generating function (mgf) and order statistics. Further, we study a new lifetime model, based on the APT family, called the alpha power exponentiated Weibull (APEW) distribution, and derive some of its structural properties. Finally, we offer some conclusions in Section 8.

The rest of the paper is outlined as follows. In Section 2, we provide important characteristics of the APT family. In Section 3, we define the APEW distribution. We obtain some of its mathematical properties in Section 4. In Section 5, we determine the maximum likelihood estimates (MLEs) of the APEW parameters. In Section 6, we investigate the performance of these estimates by means of some simulations. In Section 7 , we illustrate the potentiality of the new distribution by using two real data sets. Finally, we offer some conclusions in Section 8.

stylefancy 
The Alpha Power Transformation Family: Properties and Applications

\section{Some APT properties}

In this section, we derive some algebraic expansions to determine structural quantities of the APT family. The formulae can be implemented in R, Maple, Mathematica and Matlab packages.

\subsection{Linear representation}

First, we derive a linear representation for the pdf of $X$. For $\alpha>0, \alpha \neq 1$, equation (2) can be expanded using the power series

$$
\alpha^{z}=\sum_{k=0}^{\infty} \frac{(\log \alpha)^{k}}{k !} z^{k} .
$$

Thus, the APT family density can be expressed as

$$
f_{A P T}(x)=\frac{1}{(\alpha-1)} \sum_{k=0}^{\infty} \frac{(\log \alpha)^{k+1}}{k !} g(x) G(x)^{k} .
$$

Let $h_{k+1}(x)=(k+1) g(x) G(x)^{k}$ be the exponentiated-G (Exp-G) density with power parameter $k+1$ (for $k \geq 0$ ). Hence, the APT family density can be written as a linear combination of Exp-G densities, namely

$$
f_{A P T}(x)=\sum_{k=0}^{\infty} b_{k} h_{k+1}(x),
$$

where $b_{k}=(\log \alpha)^{k+1} /[(\alpha-1)(k+1) !]$.

Thus, some structural properties of the APT family can be determined from those of the Exp-G distribution. By integrating (5), we obtain

$$
F_{A P T}(x)=\sum_{k=0}^{\infty} b_{k} H_{k+1}(x),
$$

where $H_{k+1}(x)$ is the cdf of the Exp-G family with power parameter $k+1$.

\subsection{Moments}

Henceforth, let $Y_{k+1}$ denote the Exp-G random variable with power parameter $k+1$. The $r$ th moment of $X$, say $\mu_{r}^{\prime}=E\left(X^{r}\right)$, is obtained from equation (5) as

$$
\mu_{r}^{\prime}=\sum_{k=0}^{\infty} b_{k} E\left(Y_{k+1}^{r}\right)=\sum_{k=0}^{\infty}(k+1) b_{k} \delta_{r, k},
$$

where $\delta_{r, k}=\int_{0}^{1}\left[Q_{G}(u)\right]^{r} u^{k} d u$ and $Q_{G}(u)=G^{-1}(u)$ is the baseline quantile function (qf). 


\subsection{Generating function}

The mgf $M_{X}(t)=E\left(\mathrm{e}^{\mathrm{tX}}\right)$ of $X$ can be expressed from (5) as

$$
M_{X}(t)=\sum_{k=0}^{\infty} b_{k} M_{k+1}(t)=\sum_{k=0}^{\infty}(k+1) b_{k} \tau(t, k)
$$

where $M_{k+1}(t)$ is the mgf of $Y_{k+1}($ for $k \geq 0)$ and $\tau(t, k)=\int_{0}^{1} \exp \left[t Q_{G}(u)\right] u^{k} d u$ can be evaluated numerically from the baseline qf.

\subsection{Incomplete moments}

The main applications of the first incomplete moment are related to the mean deviations and Bonferroni and Lorenz curves, which are useful in several areas. The sth incomplete moment of $X$, say $\varphi_{s}(t)$, can be written from (5) as

$$
\varphi_{s}(t)=\int_{-\infty}^{t} x^{s} f_{A P T}(x) d x=\sum_{k=0}^{\infty} b_{k} \int_{-\infty}^{t} x^{s} h_{k+1}(x) d x .
$$

The last integral in (6) denotes the $s$ th incomplete moment of $Y_{k+1}$.

The mean deviations of $X$ about the mean, $\delta_{1}=E\left(\left|X-\mu_{1}^{\prime}\right|\right)$, and about the median, $\delta_{2}=E(|X-M|)$, are given by $\delta_{1}=2 \mu_{1}^{\prime} F_{A P T}\left(\mu_{1}^{\prime}\right)-2 \varphi_{1}\left(\mu_{1}^{\prime}\right)$ and $\delta_{2}=\mu_{1}^{\prime}-2 \varphi_{1}(M)$, respectively, where $\mu_{1}^{\prime}=E(X), M=\operatorname{Median}(X)=Q(0.5)$ is the median, $F_{A P T}\left(\mu_{1}^{\prime}\right)$ is easily evaluated from $(1)$ and $\varphi_{1}(t)$ is the first incomplete moment given by $(6)$ with $s=1$. Here, $Q(z)=G^{-1}(\log [z(\alpha-1)+1] / \log \alpha)$ is the qf of $X$.

The quantity $\varphi_{1}(t)$ follows from $(5)$ as

$$
\varphi_{1}(t)=\sum_{k=0}^{\infty} b_{k} J_{k+1}(t)=\sum_{k=0}^{\infty}(k+1) b_{k} \omega_{k}(t)
$$

where $J_{k+1}(t)=\int_{-\infty}^{t} x h_{k+1}(x) d x$ is the first incomplete moment of the Exp-G distribution and $\omega_{k}(t)=\int_{0}^{G(t)} Q_{G}(u) u^{k} d u$ can be evaluated numerically.

For a given probability $\pi$, the formula for $\varphi_{1}(t)$ can be used to construct Bonferroni and Lorenz curves given by $B(\pi)=\varphi_{1}(q) /\left(\pi \mu_{1}^{\prime}\right)$ and $L(\pi)=\varphi_{1}(q) / \mu_{1}^{\prime}$, respectively, where $q=Q(\pi)$ is the qf of $X$ evaluated at $\pi$.

\subsection{Order statistics}

Let $X_{1}, \cdots, X_{n}$ be a random sample of size $n$ from the APT distribution and $X_{(1)}, \cdots, X_{(n)}$ be the corresponding order statistics. Then, the pdf of the $r$ th order statistic $X_{r: n}$, say $f_{r: n}(x)$, is

$$
f_{r: n}(x)=\frac{f_{A P T}(x)}{\mathrm{B}(r, n-r+1)} \sum_{s=0}^{n-r}(-1)^{s}\left(\begin{array}{c}
n-r \\
s
\end{array}\right) F_{A P T}(x)^{s+r-1},
$$


where $\mathrm{B}(\cdot, \cdot)$ is the beta function. Using (1) and $(2)$, we have

$$
f_{A P T}(x) F_{A P T}(x)^{s+r-1}=\frac{\log \alpha}{(1-\alpha)^{s+r}} g(x) \alpha^{G(x)}\left[1-\alpha^{G(x)}\right]^{s+r-1} .
$$

Applying the generalized binomial expansion and the power series (3), we obtain

$$
\begin{aligned}
f_{A P T}(x) F_{A P T}(x)^{s+r-1}= & \sum_{j, k=0}^{\infty} \frac{(-1)^{j}(j+1)^{k}(\log \alpha)^{k+1}}{(1-\alpha)^{s+r} k !} \\
& \times\left(\begin{array}{c}
s+r-1 \\
j
\end{array}\right) g(x) G(x)^{k} .
\end{aligned}
$$

Combining (7) and (8), we can write

$$
f_{r: n}(x)=\sum_{k=0}^{\infty} c_{k} h_{k+1}(x)
$$

where

$$
c_{k}=\frac{(\log \alpha)^{k+1}}{(k+1) !} \sum_{j=0}^{\infty} \sum_{s=0}^{n-r} \frac{(-1)^{s+j}(j+1)^{k}}{\mathrm{~B}(j, n-j+1)(1-\alpha)^{s+r}}\left(\begin{array}{c}
n-r \\
s
\end{array}\right)\left(\begin{array}{c}
s+r-1 \\
j
\end{array}\right)
$$

and $h_{k+1}(x)$, as before, denotes the the Exp-G density with power parameter $k+1$. Thus, the density function of the APT order statistics is a linear combination of ExpG densities. Based on equation (9), we can obtain some structural properties of $X_{r: n}$ from those Exp-G properties.

\section{The APEW distribution}

The Weibull (W) distribution is a popular lifetime distribution in reliability theory. In the last few years, several researchers have developed various extensions and generalized forms of the $\mathrm{W}$ distribution to model various types of data. Among these, Mudholkar et al. (1995) and Mudholkar et al. (1996) introduced and studied the exponentiated W (EW) distribution to analyze bathtub failure data by adding an extra shape parameter to the $\mathrm{W}$ distribution.

The cdf of the EW distribution (for $x>0$ ) is

$$
G(x)=\left[1-\exp \left(-\lambda x^{\beta}\right)\right]^{\theta},
$$

where $\lambda>0$ is a scale parameter, and $\beta>0$ and $\theta>0$ are shape parameters.

The pdf corresponding to (10) is

$$
g(x)=\beta \lambda \theta x^{\beta-1} \exp \left(-\lambda x^{\beta}\right)\left[1-\exp \left(-\lambda x^{\beta}\right)\right]^{\theta-1} .
$$

We define the cdf of the APEW model, say $F(x)=F_{A P E W}(x)$, by substituting $(10)$ 
in equation (1). It is given by (for $x>0$ )

$$
F(x)=\left\{\begin{array}{cc}
\frac{1}{1-\alpha}\left\{1-\alpha^{\left[1-\exp \left(-\lambda x^{\beta}\right)\right]^{\theta}}\right\} & \text { if } \quad \alpha>0, \alpha \neq 1 \\
{\left[1-\exp \left(-\lambda x^{\beta}\right)\right]^{\theta}} & \text { if } \quad \alpha=1
\end{array}\right.
$$

The APEW pdf follows by inserting (10) and (11) in equation (2)

$$
f(x)=\left\{\begin{array}{lc}
\frac{\log (\alpha) \beta \lambda \theta x^{\beta-1}}{(\alpha-1) \exp \left(\lambda x^{\beta}\right)}\left[1-\exp \left(-\lambda x^{\beta}\right)\right]^{\theta-1} \alpha^{\left[1-\exp \left(-\lambda x^{\beta}\right)\right]^{\theta}} & \text { if } \quad \alpha>0, \alpha \neq 1 \\
\lambda \theta \beta x^{\beta-1} \exp \left(-\lambda x^{\beta}\right)\left[1-\exp \left(-\lambda x^{\beta}\right)\right]^{\theta-1} & \text { if } \quad \alpha=1 .
\end{array}\right.
$$

Henceforth, we denote by $X \sim \operatorname{APEW}(\alpha, \beta, \lambda, \theta)$ a rv having pdf (13).

The hazard rate function (hrf) of $X$ is random variable

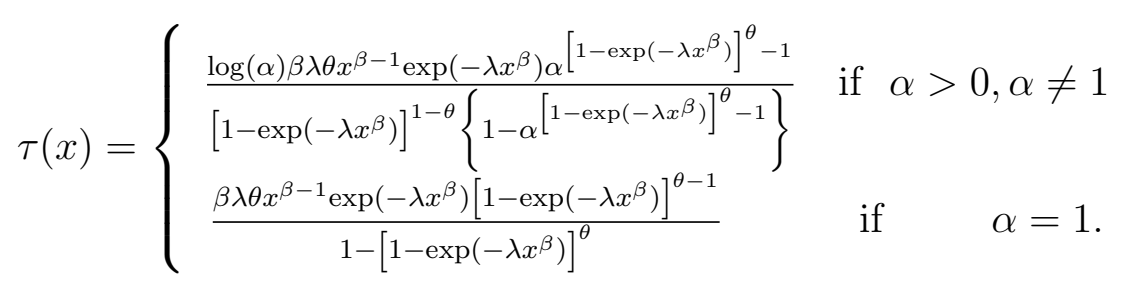

The APEW distribution is a very flexible model having at least fifteen sub-models as those listed in Table 1.

Figure 1 displays plots of the density of $X$ for some parameter values. The plots indicate that the APEW density can be reversed J-shape, left skewed, right skewed, concave down or symmetric. Figure 2 reveals that the hrf of the APEW distribution can accommodate decreasing, increasing, bathtub, unimodal and modified bathtub shapes, and thus it becomes an important model to fit real lifetime data.

\section{APEW properties}

As in Section 2, we provide algebraic expansions to determine some structural quantities of the APEW distribution.

\subsection{Linear representation}

We derive a linear representations for the pdf of $X$. First, we expand (13) when $\alpha>0$ $(\alpha \neq 1)$ using the power series (3). The case $\alpha=1$ is discussed by Nadarajah et al. (2013). We can write

$$
\alpha^{\left[1-\exp \left(-\lambda x^{\beta}\right)\right]^{\theta}}=\sum_{k=0}^{\infty} \frac{(\log \alpha)^{k}}{k !}\left[1-\exp \left(-\lambda x^{\beta}\right)\right]^{k \theta} .
$$

Thus, the APEW density can be expressed as

$$
f(x)=\frac{\lambda \theta \beta x^{\beta-1}}{(\alpha-1) \exp \left(\lambda x^{\beta}\right)} \sum_{k=0}^{\infty} \frac{(\log \alpha)^{k+1}}{k !}\left[1-\exp \left(-\lambda x^{\beta}\right)\right]^{(k+1) \theta-1} .
$$



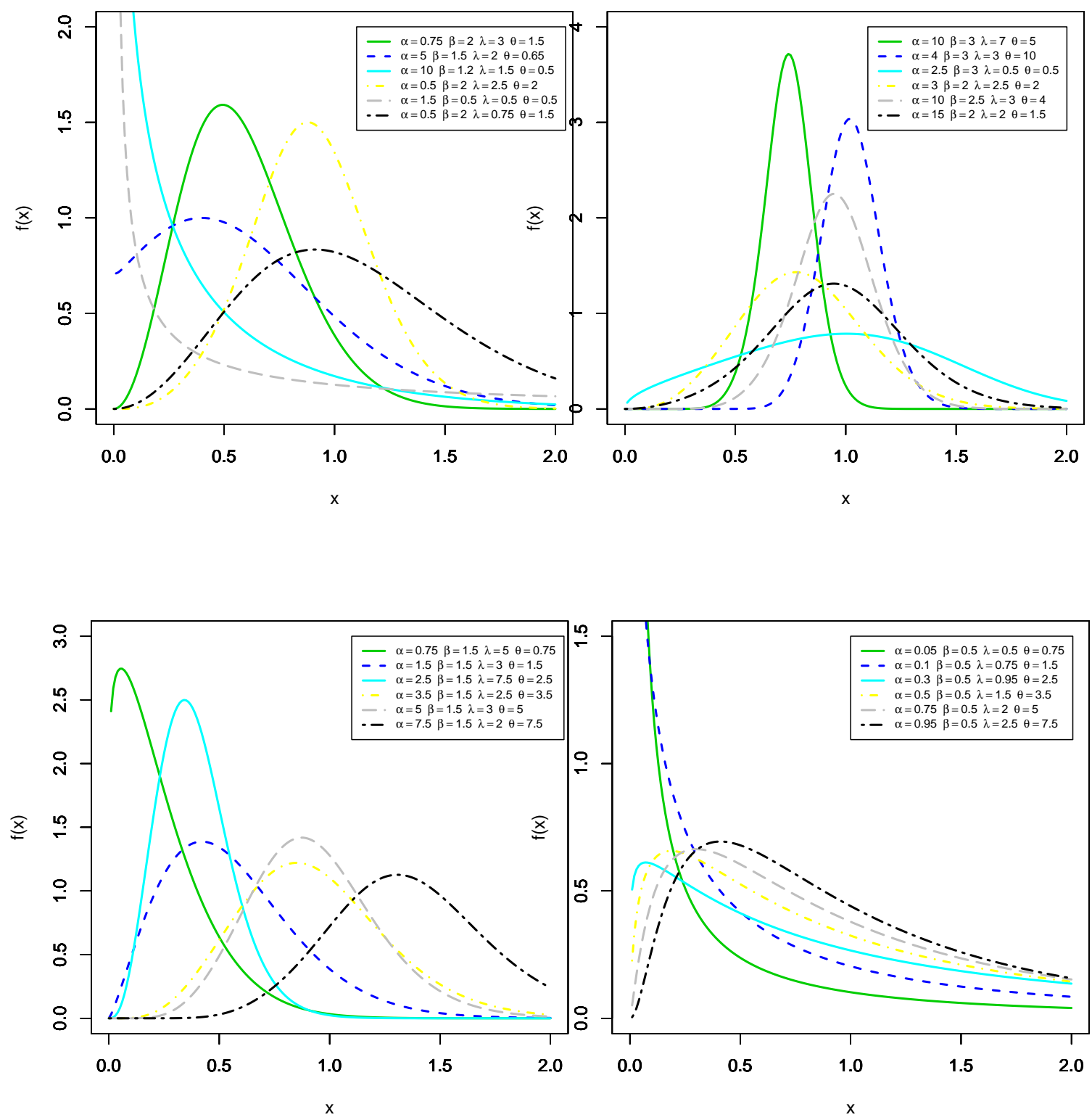

Figure 1: Plots of the APEW density function for some parameter values. 
Mohamed E. Mead, Gauss M. Cordeiro, Ahmed Z. Afify, Hazem Al-Mofleh

Table 1: Sub-models of the APEW distribution

\begin{tabular}{llccccc}
\hline \hline No. & Reduced Model & $\alpha$ & $\beta$ & $\lambda$ & $\theta$ & Author \\
\hline 1 & APER & & 2 & & & New \\
2 & APEEx & & 1 & & & New \\
3 & APIIEW & & & 1 & & New \\
4 & APW & & & & 1 & (Nassar et al., 2017) \\
5 & APR & & 2 & & 1 & - \\
6 & APEx & & 1 & & 1 & (Mahdavi and Kundu, 2017) \\
7 & APIW & & & 1 & 1 & \\
8 & EW & 1 & & & & \\
9 & ER & 1 & 2 & & & \\
10 & EEx & 1 & 1 & & & (Weibull, 1951) \\
11 & IIEW & 1 & & 1 & & - \\
12 & IIW & 1 & & & 1 & \\
13 & Ex & 1 & 1 & 1 & & (Rayleigh, 1880) \\
14 & R & 1 & 2 & 1 & & \\
15 & IW & 1 & & 1 & 1 & \\
& & & & & &
\end{tabular}

Abbreviations: $\mathrm{E}=$ Exponentiated, $\mathrm{R}=$ Rayleigh, $\mathrm{Ex}=$ Exponential, $\mathrm{II}=$ two-parameter, $\mathrm{I}=$ one parameter.
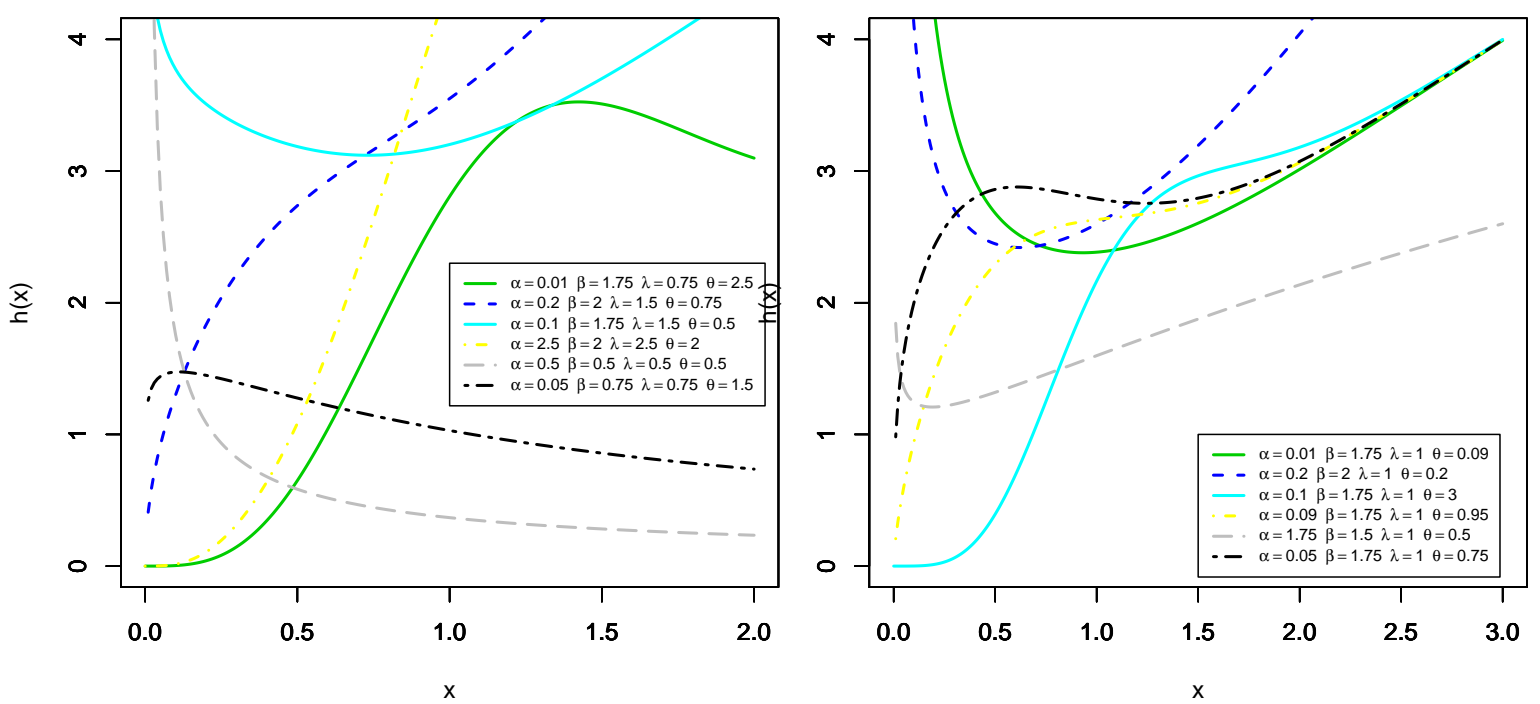

Figure 2: Plots of the APEW hrf for some parameter values. 
The Alpha Power Transformation Family: Properties and Applications

Consider the generalized binomial expansion given by

$$
(1-z)^{b-1}=\sum_{j=0}^{\infty}(-1)^{j}\left(\begin{array}{c}
b-1 \\
j
\end{array}\right) z^{j},|z|<1, b>0 .
$$

Using expansion (15) in (14) and after some algebra, the pdf of $X$ reduces to

$$
f(x)=\frac{\beta \lambda \theta x^{\beta-1}}{(\alpha-1)} \sum_{k, j=0}^{\infty} \frac{(-1)^{j}(\log \alpha)^{k+1}}{k !}\left(\begin{array}{c}
(k+1) \theta-1 \\
j
\end{array}\right) \exp \left[-(j+1) \lambda x^{\beta}\right] .
$$

Hence, the pdf of $X$ can be written as (which also holds when $\alpha=1$ )

$$
f(x)=\sum_{j=0}^{\infty} b_{j} \pi_{(j+1) \lambda, \beta}(x)
$$

where

$$
b_{j}=\left\{\begin{array}{lr}
\sum_{k=0}^{\infty} \frac{(-1)^{j} \theta(\log \alpha)^{k+1}}{(\alpha-1)(j+1) k !}\left(\begin{array}{c}
(k+1) \theta-1 \\
j
\end{array}\right) & \text { if } \alpha>0, \alpha \neq 1 \\
\frac{\theta(-1)^{j}}{(j+1)}\left(\begin{array}{c}
\theta-1 \\
j
\end{array}\right) & \text { if } \alpha=1
\end{array} \quad\right. \text { (Nadarajah et al., 2013), }
$$

and $\pi_{(j+1) \lambda, \beta}(x)$ is the $\mathrm{W}$ density function with scale parameter $(j+1) \lambda$ and shape parameter $\beta$. Equation (16) indicates that the APEW pdf is a linear combination of $\mathrm{W}$ densities. So, several of its structural properties can be determined from those of the $\mathrm{W}$ distribution.

Henceforth, let $Z$ be a $\mathrm{W}$ random variable with positive shape parameter $\beta$ and positive scale parameter $\lambda$. Then, the pdf of $Z$ is

$$
\pi(z ; \lambda, \beta,)=\lambda \beta z^{\beta-1} \exp \left(-\lambda z^{\beta}\right) .
$$

The $r$ th ordinary and incomplete moments of $Z$ are

$$
\mu_{r, Z}^{\prime}=\lambda^{-r / \beta} \Gamma(1+r / \beta) \text { and } \varphi_{r, Z}(t)=\lambda^{-r / \beta} \gamma\left(1+r / \beta, \lambda t^{\beta}\right)
$$

respectively, where $\gamma(a, t)=\int_{0}^{t} x^{a-1} \mathrm{e}^{-x} d x$ is the lower incomplete gamma function.

\subsection{Quantile and generating functions}

First, we obtain the qf of $X$, say $Q(u)$, by inverting (12). For $\alpha>0, \alpha \neq 1$, we have

$$
Q(u)=\left\{-\lambda^{-1} \log \left[1-\left(\frac{\log [1+(\alpha-1) u]}{\log \alpha}\right)^{1 / \theta}\right]\right\}^{1 / \beta}, 0<u<1
$$

Simulating the APEW random variable is straightforward. If $U$ is a uniform variable on the unit interval $(0,1)$, then the random variable $X=Q(U)$ has pdf (13). 
Mohamed E. Mead, Gauss M. Cordeiro, Ahmed Z. Afify, Hazem Al-Mofleh

Second, the mgf of $Z$ follows from Nadarajah et al. (2013) as

$$
M_{Z}(t ; \lambda, \beta)=\lambda \beta \int_{0}^{\infty} \exp (t x) x^{\beta-1} \exp \left(-\lambda x^{\beta}\right) d x
$$

By expanding $\exp (t x)$ and calculating the integral, we obtain

$$
M_{Z}(t ; \lambda, \beta)=\sum_{m=0}^{\infty} \frac{t^{m}}{m ! \lambda^{m / \beta}} \Gamma(1+m / \beta) .
$$

We use the Wright generalized hypergeometric function given by

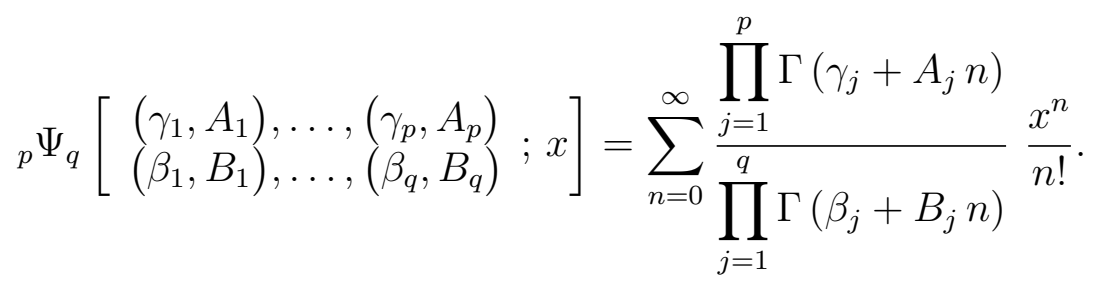

Then, we can rewrite $M_{Z}(t ; \lambda, \beta)$ as

$$
M_{Z}(t ; \lambda, \beta)={ }_{1} \Psi_{0}\left[\begin{array}{c}
\left(1,-\beta^{-1}\right) \\
-
\end{array}\right] \lambda t .
$$

Combining the last expression and equation (16), we obtain the mgf of $X$ as

$$
M_{X}(t)=\sum_{j=0}^{\infty} b_{j 1} \Psi_{0}\left[\begin{array}{c}
\left(1,-\beta^{-1}\right) \\
-
\end{array} ;(j+1) \lambda t\right] .
$$

\subsection{Moments}

The $s$ th ordinary moment of $X$, say $\mu_{s}^{\prime}$, can be expressed from (16) as

$$
\mu_{s}^{\prime}=\sum_{j=0}^{\infty} b_{j}[(j+1) \lambda]^{-s / \beta} \Gamma(1+s / \beta) .
$$

The sth incomplete moment of $X$ follows from (16) as

$$
\varphi_{s}(t)=\sum_{j=0}^{\infty} b_{j} \int_{0}^{t} x^{s} \pi_{(j+1) \lambda, \beta}(x) d x .
$$

Then, $\varphi_{s}(t)$ has the simple form

$$
\varphi_{s}(t)=\sum_{j=0}^{\infty} b_{j}[(j+1) \lambda]^{-s / \beta} \gamma\left(1+s / \beta,(j+1) \lambda t^{\beta}\right) \text {. }
$$




\subsection{Order statistics}

Let $X_{1}, \cdots, X_{n}$ be a random sample of size $n$ from the APEW distribution and $X_{(1)}, \cdots, X_{(n)}$ be the order statistics in the sample. Then, the pdf of the $r$ th order statistic $X_{r: n}$, say $f_{r: n}(x)$. Using the generalized binomial expansion and the power series (3), we obtain

$$
F(x)^{s+r-1}=v \sum_{w, k=0}^{\infty} \frac{(-1)^{w}(\log \alpha)^{k}}{k !}\left(\begin{array}{c}
s+r-1 \\
w
\end{array}\right)\left[1-\exp \left(-\lambda x^{\beta}\right)\right]^{\theta k w},
$$

where $v=[1 /(1-\alpha)]^{s+r-1}$.

Further, we can write

$$
\begin{aligned}
f(x) F(x)^{s+r-1}= & \frac{v \lambda \theta \beta x^{\beta-1}}{\exp \left(-\lambda x^{\beta}\right)} \sum_{w, k=0}^{\infty} \frac{(-1)^{w}(\log \alpha)^{k+1}}{(\alpha-1) k !}\left(\begin{array}{c}
s+r-1 \\
w
\end{array}\right) \\
& \times \alpha^{\left[1-\exp \left(-\lambda x^{\beta}\right)\right]^{\theta}}\left[1-\exp \left(-\lambda x^{\beta}\right)\right]^{\theta(k w+1)-1} .
\end{aligned}
$$

By using (3) and (15) and after some simplification, we have

$$
\begin{aligned}
f(x) F(x)^{s+r-1}= & \sum_{w, k, j, m=0}^{\infty} \frac{(-1)^{w+m} v \theta(\log \alpha)^{k+w+1}}{(\alpha-1) k ! j !}\left(\begin{array}{c}
s+r-1 \\
w
\end{array}\right) \\
& \times\left(\begin{array}{c}
\theta(k w+j+1)-1 \\
m
\end{array}\right) \lambda \beta x^{\beta-1} \exp \left[-(m+1) \lambda x^{\beta}\right] .
\end{aligned}
$$

Combining the last equation and (7), we obtain

$$
f_{r: n}(x)=\sum_{m=0}^{\infty} p_{m} \pi_{(m+1) \lambda, \beta}(x)
$$

where

$$
\begin{aligned}
p_{m}= & \frac{v \theta}{(\alpha-1)(m+1)} \sum_{w, k, j=0}^{\infty} \sum_{s=0}^{n-r} \frac{(-1)^{s+w+m}(\log \alpha)^{k+w+1}}{\mathrm{~B}(j, n-j+1) k ! j !} \\
& \times\left(\begin{array}{c}
n-r \\
s
\end{array}\right)\left(\begin{array}{c}
s+r-1 \\
w
\end{array}\right)\left(\begin{array}{c}
\theta(k w+j+1)-1 \\
m
\end{array}\right)
\end{aligned}
$$

and $\pi_{(m+1) \lambda, \beta}(x)$, as in Section 4.1 , denotes the $\mathrm{W}$ density function with shape parameter $\beta$ and scale parameter $(m+1) \lambda$. Thus, the density function of the APEW order statistics is a linear combination of the $W$ densities. Based on equation (18), we can obtain some structural properties of $X_{r: n}$ from those $\mathrm{W}$ properties. 


\section{Maximum likelihood estimation}

The maximum likelihood estimators (MLEs) enjoy desirable properties and can be used for constructing confidence intervals for the model parameters. Further, the normal approximation for these estimators in large samples can be easily handled, either analytically or numerically. Let $x_{1}, \cdots, x_{n}$ be a random sample from the APEW distribution with unknown parameter vector $\varphi=(\alpha, \beta, \lambda, \theta)^{T}$.

The $\log$-likelihood function for $\varphi$, say $\ell=\ell(\varphi)$, is

$$
\begin{aligned}
\ell= & n \log \left(\frac{\log \alpha}{\alpha-1}\right)+n \log (\beta \lambda \theta)+(\beta-1) \sum_{i=1}^{n} \log x_{i}-\lambda \sum_{i=1}^{n} x_{i}^{\beta} \\
& +(\theta-1) \sum_{i=1}^{n} \log \left[1-\exp \left(-\lambda x_{i}^{\beta}\right)\right]+\sum_{i=1}^{n}\left[1-\exp \left(-\lambda x_{i}^{\beta}\right)\right]^{\theta} \log \alpha .
\end{aligned}
$$

The log-likelihood (19) can be maximized numerically using the R (optim function), SAS (PROC NLMIXED), Ox program (sub-routine MaxBFGS), among others.

The score vector $\mathbf{U}(\varphi)=\frac{\partial \ell}{\partial \varphi}=\left(\frac{\partial \ell}{\partial \alpha}, \frac{\partial \ell}{\partial \beta}, \frac{\partial \ell}{\partial \lambda}, \frac{\partial \ell}{\partial \theta}\right)^{T}$ is available from the corresponding author under request. For interval estimation of the model parameters, we require the $4 \times 4$ observed information matrix $J(\varphi)=\left\{J_{r s}\right\}$ for $r, s=\alpha, \beta, \lambda, \theta$. Under standard regularity conditions, the multivariate normal $N_{4}\left(0, J(\widehat{\varphi})^{-1}\right)$ distribution can be used to construct approximate confidence intervals for the parameters. Here, $J(\widehat{\varphi})$ is the total observed information matrix evaluated at $\widehat{\varphi}$.

\section{Simulation study}

We now present some simulation results to investigate the behavior of the MLEs in terms of the sample size $n$. All simulations are performed using the $\mathrm{R}$ programming language (R Core Team, 2019). We generate 2, 000 random samples from the APEW distribution using equation (17) with sample sizes $n=250$ and $n=750$. The true values of the parameters are chosen as: $\alpha=(0.65,1.6,3), \beta=(0.7,1.5,4), \lambda=$ $(0.75,1.7,5)$ and $\theta=(0.8,1.8,6)$. For each sample size and each parameter combination, the average of the MLEs and mean square errors (MSEs) are computed. Tables 2 and 3 provide these results thus indicating that the estimators are stable and quite close to the true parameter values for both sample sizes. Furthermore, the mean squared errors decrease when $n$ increases for all cases in agreement with first-order asymptotic theory.

\section{Real data applications}

In this section, we provide two applications to real data sets to illustrate the importance and flexibility of the APEW distribution.

The first data set corresponds to the exceedances of flood peaks (in $\mathrm{m} 3 / \mathrm{s}$ ) of the Wheaton River near Carcross in Yukon Territory, Canada. These data consist of 72 exceedances for the years 1958-1984, rounded to one decimal place. They have been analyzed by Choulakian and Stephens (2001). The second data set is obtained from Smith and Naylor (1987). The data are the strengths of $1.5 \mathrm{~cm}$ glass fibres measured 
The Alpha Power Transformation Family: Properties and Applications

Table 2: Average values of the MLEs and MSEs $(n=250)$

\begin{tabular}{|c|c|c|c|c|c|c|c|c|c|c|c|}
\hline \multicolumn{4}{|c|}{ Parameters } & \multicolumn{4}{|c|}{ MLE } & \multicolumn{4}{|c|}{ MSE } \\
\hline$\alpha$ & $\beta$ & $\lambda$ & $\theta$ & $\widehat{\alpha}$ & $\widehat{\beta}$ & $\widehat{\lambda}$ & $\widehat{\theta}$ & $\widehat{\alpha}$ & $\widehat{\beta}$ & $\widehat{\lambda}$ & $\widehat{\theta}$ \\
\hline \multirow[t]{27}{*}{0.56000} & \multirow[t]{9}{*}{0.70000} & \multirow[t]{3}{*}{0.75000} & 0.80000 & 0.58401 & 0.72924 & 0.75565 & 0.82485 & 0.00009 & 0.00010 & 0.00022 & 0.00025 \\
\hline & & & 1.80000 & 0.57651 & 0.71598 & 0.78023 & 1.95826 & 0.00008 & 0.00008 & 0.00030 & 0.00249 \\
\hline & & & 6.00000 & 0.57013 & 0.71476 & 0.80646 & 7.42377 & 0.00007 & 0.00009 & 0.00054 & 0.15047 \\
\hline & & \multirow[t]{3}{*}{1.70000} & 0.80000 & 0.57280 & 0.73274 & 1.70281 & 0.82471 & 0.00009 & 0.00011 & 0.00032 & 0.00026 \\
\hline & & & 1.80000 & 0.57927 & 0.71659 & 1.71914 & 1.92852 & 0.00008 & 0.00007 & 0.00043 & 0.00210 \\
\hline & & & 6.00000 & 0.57378 & 0.72376 & 1.72928 & 7.30097 & 0.00007 & 0.00009 & 0.00105 & 0.16359 \\
\hline & & \multirow[t]{3}{*}{5.00000} & 0.80000 & 0.57381 & 0.72487 & 5.22544 & 0.84170 & 0.00009 & 0.00010 & 0.00199 & 0.00028 \\
\hline & & & 1.80000 & 0.57297 & 0.72354 & 5.13358 & 1.93533 & 0.00007 & 0.00009 & 0.00063 & 0.00249 \\
\hline & & & 6.00000 & 0.57174 & 0.71811 & 5.08595 & 7.25462 & 0.00007 & 0.00008 & 0.00075 & 0.11104 \\
\hline & \multirow{9}{*}{1.50000} & \multirow[t]{3}{*}{0.75000} & 0.80000 & 0.57463 & 1.56500 & 0.75706 & 0.82776 & 0.00009 & 0.00047 & 0.00024 & 0.00027 \\
\hline & & & 1.80000 & 0.57338 & 1.55470 & 0.76474 & 1.92467 & 0.00007 & 0.00038 & 0.00030 & 0.00250 \\
\hline & & & 6.00000 & 0.57857 & 1.53531 & 0.79774 & 7.25838 & 0.00007 & 0.00037 & 0.00050 & 0.11414 \\
\hline & & \multirow[t]{3}{*}{1.70000} & 0.80000 & 0.57952 & 1.57475 & 1.69141 & 0.81366 & 0.00009 & 0.00045 & 0.00030 & 0.00024 \\
\hline & & & 1.80000 & 0.57344 & 1.54226 & 1.72059 & 1.94167 & 0.00007 & 0.00036 & 0.00048 & 0.00244 \\
\hline & & & 6.00000 & 0.57077 & 1.52626 & 1.76697 & 7.56567 & 0.00007 & 0.00038 & 0.00104 & 0.24379 \\
\hline & & \multirow[t]{3}{*}{5.00000} & 0.80000 & 0.57336 & 1.56878 & 5.22230 & 0.82052 & 0.00009 & 0.00042 & 0.00176 & 0.00024 \\
\hline & & & 1.80000 & 0.57122 & 1.54751 & 5.12895 & 1.93605 & 0.00007 & 0.00038 & 0.00056 & 0.00242 \\
\hline & & & 6.00000 & 0.57245 & 1.53938 & 5.09143 & 7.53353 & 0.00007 & 0.00041 & 0.00085 & 0.25313 \\
\hline & \multirow[t]{9}{*}{4.00000} & \multirow[t]{3}{*}{0.75000} & 0.80000 & 0.57219 & 4.16967 & 0.75925 & 0.83052 & 0.00009 & 0.00325 & 0.00023 & 0.00027 \\
\hline & & & 1.80000 & 0.57458 & 4.12920 & 0.76701 & 1.92281 & 0.00008 & 0.00258 & 0.00028 & 0.00221 \\
\hline & & & 6.00000 & 0.57927 & 4.08478 & 0.80246 & 7.26152 & 0.00007 & 0.00265 & 0.00049 & 0.09622 \\
\hline & & \multirow[t]{3}{*}{1.70000} & 0.80000 & 0.57459 & 4.16901 & 1.70529 & 0.82503 & 0.00009 & 0.00311 & 0.00030 & 0.00025 \\
\hline & & & 1.80000 & 0.57548 & 4.12398 & 1.71225 & 1.91913 & 0.00008 & 0.00255 & 0.00045 & 0.00213 \\
\hline & & & 6.00000 & & 4.08415 & 1.7 & & 07 & 0.00285 & 0.00110 & 0.23230 \\
\hline & & 5.00000 & 0.80000 & 0.57847 & 4.18441 & 5.22552 & 0.82070 & 0.00009 & 0.00313 & 0.00193 & 0.00025 \\
\hline & & & 1.80000 & 0.57155 & 4.12848 & 5.12665 & 1.93621 & 0.00007 & 0.00269 & 0.00057 & 0.00255 \\
\hline & & & 6.00000 & 0.57108 & 4.06899 & 5.10199 & 7.35618 & 0.00007 & 0.00260 & 0.00073 & 0.10496 \\
\hline 1.60000 & 0.70000 & 0.75000 & 0.80000 & 1.64840 & 0.72023 & 0.76491 & 0.83869 & 0.00084 & 0.00007 & 0.00023 & 0.00028 \\
\hline & & & 1.80000 & 1.66076 & 0.71617 & 0.77357 & 1.94477 & 0.00073 & 0.00007 & 0.00028 & 0.00236 \\
\hline & & & 6.00000 & 1.63335 & 0.71350 & 0.81196 & 7.69644 & 0.00059 & 0.00008 & 0.00059 & 0.22431 \\
\hline & & 1.70000 & 0.80000 & 1.66667 & 0.72743 & 1.69890 & 0.82663 & 0.00099 & 0.00008 & 0.00035 & 0.00028 \\
\hline & & & 1.80000 & 1.64811 & 0.71856 & 1.71389 & 1.94203 & 0.00069 & 0.00007 & 0.00051 & 0.00270 \\
\hline & & & 6.00000 & 1.64034 & 0.71551 & 1.74962 & 7.47891 & 0.00058 & 0.00008 & 0.00104 & 0.20192 \\
\hline & & 5.00000 & 0.80000 & 1.66382 & 0.73020 & 5.15737 & 0.82237 & 0.00085 & 0.00008 & 0.00085 & 0.00026 \\
\hline & & & 1.80000 & 1.66402 & 0.71626 & 5.10231 & 1.98083 & 0.00077 & 0.00007 & 0.00041 & 0.00311 \\
\hline & & & 6.00000 & 1.64576 & 0.71828 & 5.06707 & 7.38368 & 0.00065 & 0.00008 & 0.00090 & 0.18309 \\
\hline & 1.50000 & 0.75000 & 0.80000 & 1.67235 & 1.54878 & 0.75840 & 0.82873 & 0.00092 & 0.00035 & 0.00022 & 0.00026 \\
\hline & & & 1.80000 & 1.65184 & 1.52821 & 0.78313 & 1.97376 & 0.00067 & 0.00032 & 0.00030 & 0.00254 \\
\hline & & & 6.00000 & 1.64561 & 1.52566 & 0.81115 & 7.67203 & 0.00058 & 0.00034 & 0.00055 & 0.22491 \\
\hline & & 1.70000 & 0.80000 & 1.67399 & 1.55506 & 1.70437 & 0.83160 & 0.00092 & 0.00038 & 0.00035 & 0.00028 \\
\hline & & & 1.80000 & 1.64187 & 1.54229 & 1.71452 & 1.94099 & 0.00069 & 0.00034 & 0.00052 & 0.00251 \\
\hline & & & 6.00000 & 1.64543 & 1.51948 & 1.77492 & 7.65341 & 0.00062 & 0.00035 & 0.00106 & 0.17676 \\
\hline & & 5.00000 & 0.80000 & 1.66196 & 1.55537 & 5.14854 & 0.82730 & 0.00088 & 0.00035 & 0.00086 & 0.00027 \\
\hline & & & 1.80000 & 1.64818 & 1.54043 & 5.10380 & 1.94816 & 0.00069 & 0.00033 & 0.00040 & 0.00240 \\
\hline & & & 6.00000 & 1.64013 & 1.52557 & 5.10240 & 7.84336 & 0.00059 & 0.00036 & 0.00103 & 0.25000 \\
\hline & 4.00000 & 0.75000 & 0.80000 & 1.68478 & 4.12256 & 0.76092 & 0.83105 & 0.00099 & 0.00251 & 0.00022 & 0.00026 \\
\hline & & & 1.80000 & 1.64749 & 4.09309 & 0.77603 & 1.95905 & 0.00071 & 0.00220 & 0.00030 & 0.00266 \\
\hline & & & 6.00000 & 1.64444 & 4.08434 & 0.80333 & 7.47007 & 0.00059 & 0.00256 & 0.00053 & 0.15778 \\
\hline & & 1.70000 & 0.80000 & 1.68048 & 4.12287 & 1.70970 & 0.83640 & 0.00096 & 0.00267 & 0.00036 & 0.00029 \\
\hline & & & 1.80000 & 1.66760 & 4.09532 & 1.71929 & 1.94972 & 0.00077 & 0.00207 & 0.00050 & 0.00262 \\
\hline & & & 6.00000 & 1.63628 & 4.05717 & 1.77034 & 7.66897 & 0.00058 & 0.00247 & 0.00109 & 0.17853 \\
\hline & & 5.00000 & 0.80000 & 1.65761 & 4.16783 & 5.15147 & 0.82366 & 0.00090 & 0.00275 & 0.00094 & 0.00027 \\
\hline & & & 1.80000 & 1.64354 & 4.09131 & 5.09865 & 1.96322 & 0.00070 & 0.00220 & 0.00041 & 0.00294 \\
\hline & & & 6.00000 & 1.64536 & 4.07410 & 5.07916 & 7.43310 & 0.00061 & 0.00245 & 0.00090 & 0.12411 \\
\hline
\end{tabular}

Pak.j.stat.oper.res. Vol.XV No.III 2019 pp525-545 
Mohamed E. Mead, Gauss M. Cordeiro, Ahmed Z. Afify, Hazem Al-Mofleh

Table 2: Average values of the MLEs and MSEs $(n=250)$ (Continued)

\begin{tabular}{|c|c|c|c|c|c|c|c|c|c|c|c|}
\hline \multicolumn{4}{|c|}{ Parameters } & \multicolumn{4}{|c|}{ MLE } & \multicolumn{4}{|c|}{ MSE } \\
\hline$\alpha$ & $\beta$ & $\lambda$ & $\theta$ & $\widehat{\alpha}$ & $\widehat{\beta}$ & $\hat{\lambda}$ & $\widehat{\theta}$ & $\widehat{\alpha}$ & $\widehat{\beta}$ & $\hat{\lambda}$ & $\widehat{\theta}$ \\
\hline \multirow[t]{27}{*}{3.00000} & 0.70000 & 0.75000 & 0.80000 & 3.17661 & 0.71744 & 0.76782 & 0.84205 & 0.00421 & 0.00007 & 0.00023 & 0.00029 \\
\hline & & & 1.80000 & 3.10501 & 0.71786 & 0.77310 & 1.96577 & 0.00284 & 0.00007 & 0.00030 & 0.00304 \\
\hline & & & 6.00000 & 3.10440 & 0.71277 & 0.80601 & 7.53924 & 0.00242 & 0.00007 & 0.00052 & 0.14920 \\
\hline & & 1.70000 & 0.80000 & 3.15046 & 0.72095 & 1.70410 & 0.83414 & 0.00381 & 0.00007 & 0.00036 & 0.00030 \\
\hline & & & 1.80000 & 3.12193 & 0.71176 & 1.73405 & 1.99457 & 0.00301 & 0.00006 & 0.00055 & 0.00326 \\
\hline & & & 6.00000 & 3.09880 & 0.70692 & 1.78210 & 7.77581 & 0.00248 & 0.00008 & 0.00112 & 0.15367 \\
\hline & & 5.00000 & 0.80000 & 3.15096 & 0.72502 & 5.13415 & 0.83074 & 0.00386 & 0.00007 & 0.00069 & 0.00027 \\
\hline & & & 1.80000 & 3.07676 & 0.71463 & 5.07765 & 1.96149 & 0.00272 & 0.00006 & 0.00039 & 0.00276 \\
\hline & & & 6.00000 & 3.10512 & 0.70828 & 5.10491 & 8.04785 & 0.00243 & 0.00007 & 0.00109 & 0.70358 \\
\hline & 1.50000 & 0.75000 & 0.80000 & 3.17441 & 1.54911 & 0.75455 & 0.82795 & 0.00394 & 0.00031 & 0.00022 & 0.00028 \\
\hline & & & 1.80000 & 3.11605 & 1.52277 & 0.78903 & 2.01177 & 0.00307 & 0.00030 & 0.00033 & 0.00347 \\
\hline & & & 6.00000 & 3.08578 & 1.52893 & 0.81021 & 7.75753 & 0.00220 & 0.00036 & 0.00056 & 0.22717 \\
\hline & & 1.70000 & 0.80000 & 3.15020 & 1.54500 & 1.70895 & 0.83748 & 0.00385 & 0.00034 & 0.00036 & 0.00028 \\
\hline & & & 1.80000 & 3.14651 & 1.53072 & 1.72202 & 1.96454 & 0.00299 & 0.00029 & 0.00053 & 0.00284 \\
\hline & & & 6.00000 & 3.10122 & 1.52555 & 1.76311 & 7.79205 & 0.00237 & 0.00033 & 0.00112 & 0.29415 \\
\hline & & 5.00000 & 0.80000 & 3.10999 & 1.55596 & 5.12709 & 0.82987 & 0.00358 & 0.00034 & 0.00068 & 0.00029 \\
\hline & & & 1.80000 & 3.09774 & 1.53436 & 5.08702 & 1.98954 & 0.00279 & 0.00031 & 0.00041 & 0.00367 \\
\hline & & & 6.00000 & 3.11674 & 1.52673 & 5.06868 & 7.49895 & 0.00250 & 0.00032 & 0.00102 & 0.15401 \\
\hline & 4.00000 & 0.75000 & 0.80000 & 3.14051 & 4.12495 & 0.76016 & 0.83470 & 0.00383 & 0.00240 & 0.00023 & 0.00029 \\
\hline & & & 1.80000 & 3.13158 & 4.07219 & 0.78199 & 1.98494 & 0.00284 & 0.00204 & 0.00030 & 0.00289 \\
\hline & & & 6.00000 & 3.09314 & 4.08376 & 0.80819 & 7.73416 & 0.00227 & 0.00256 & 0.00056 & 0.21193 \\
\hline & & 1.70000 & 0.80000 & 3.10761 & 4.14717 & 1.69865 & 0.83078 & 0.00357 & 0.00240 & 0.00037 & 0.00029 \\
\hline & & & 1.80000 & 3.08799 & 4.09647 & 1.71759 & 1.95454 & 0.00278 & 0.00212 & 0.00053 & 0.00276 \\
\hline & & & 6.00000 & 3.10799 & 4.05552 & 1.76892 & 7.83707 & 0.00254 & 0.00242 & 0.00114 & 0.32540 \\
\hline & & 5.00000 & 0.80000 & 3.14735 & 4.12548 & 5.11972 & 0.83290 & 0.00406 & 0.00215 & 0.00060 & 0.00028 \\
\hline & & & 1.80000 & 3.10411 & 4.10111 & 5.08678 & 1.96460 & 0.00288 & 0.00216 & 0.00039 & 0.00297 \\
\hline & & & 6.00000 & 3.09573 & 4.06645 & 5.08304 & 7.55694 & 0.00241 & 0.00221 & 0.00101 & 0.16904 \\
\hline
\end{tabular}


The Alpha Power Transformation Family: Properties and Applications

Table 3: Average values of the MLEs and MSEs $(n=750)$

\begin{tabular}{|c|c|c|c|c|c|c|c|c|c|c|c|}
\hline \multicolumn{4}{|c|}{ Parameters } & \multicolumn{4}{|c|}{ MLE } & \multicolumn{4}{|c|}{ MSE } \\
\hline$\alpha$ & $\beta$ & $\lambda$ & $\theta$ & $\widehat{\alpha}$ & $\widehat{\beta}$ & $\hat{\lambda}$ & $\widehat{\theta}$ & $\widehat{\alpha}$ & $\widehat{\beta}$ & $\widehat{\lambda}$ & $\widehat{\theta}$ \\
\hline \multirow[t]{27}{*}{0.56000} & 0.70000 & 0.75000 & 0.80000 & 0.56334 & 0.71030 & 0.75187 & 0.80846 & 0.00001 & 0.00001 & 0.00002 & 0.00002 \\
\hline & & & 1.80000 & 0.56506 & 0.70239 & 0.76358 & 1.85524 & 0.00001 & 0.00001 & 0.00003 & 0.00018 \\
\hline & & & 6.00000 & 0.56632 & 0.70226 & 0.77306 & 6.41265 & 0.00001 & 0.00001 & 0.00005 & 0.00562 \\
\hline & & 1.70000 & 0.80000 & 0.56472 & 0.70851 & 1.70541 & 0.81033 & 0.00001 & 0.00001 & 0.00003 & 0.00002 \\
\hline & & & 1.80000 & 0.56306 & 0.70747 & 1.70267 & 1.83328 & 0.00001 & 0.00001 & 0.00005 & 0.00018 \\
\hline & & & 6.00000 & 0.56429 & 0.70216 & 1.72816 & 6.44126 & 0.00001 & 0.00001 & 0.00010 & 0.00556 \\
\hline & & 5.00000 & 0.80000 & 0.56613 & 0.70759 & 5.05787 & 0.80952 & 0.00001 & 0.00001 & 0.00011 & 0.00002 \\
\hline & & & 1.80000 & 0.56617 & 0.70367 & 5.03113 & 1.84622 & 0.00001 & 0.00001 & 0.00004 & 0.00017 \\
\hline & & & 6.00000 & 0.56387 & 0.70545 & 5.02701 & 6.34496 & 0.00001 & 0.00001 & 0.00007 & 0.00545 \\
\hline & 1.50000 & 0.75000 & 0.80000 & 0.56465 & 1.51320 & 0.75766 & 0.81388 & 0.00001 & 0.00004 & 0.00003 & 0.00003 \\
\hline & & & 1.80000 & 0.56511 & 1.51365 & 0.75625 & 1.83792 & 0.00001 & 0.00003 & 0.00003 & 0.00018 \\
\hline & & & 6.00000 & 0.56285 & 1.51134 & 0.76530 & 6.33344 & 0.00001 & 0.00004 & 0.00005 & 0.00523 \\
\hline & & 1.70000 & 0.80000 & 0.56694 & 1.51627 & 1.70318 & 0.80951 & 0.00001 & 0.00004 & 0.00003 & 0.00002 \\
\hline & & & 1.80000 & 0.56463 & 1.51561 & 1.70527 & 1.84000 & 0.00001 & 0.00004 & 0.00005 & 0.00019 \\
\hline & & & 6.00000 & 0.56520 & 1.51692 & 1.70745 & 6.29775 & 0.00001 & 0.00004 & 0.00010 & 0.00537 \\
\hline & & 5.00000 & 0.80000 & 0.56603 & 1.52823 & 5.07371 & 0.80132 & 0.00001 & 0.00004 & 0.00011 & 0.00002 \\
\hline & & & 1.80000 & 0.56382 & 1.51461 & 5.03955 & 1.84002 & 0.00001 & 0.00004 & 0.00005 & 0.00019 \\
\hline & & & 6.00000 & 0.56523 & 1.51110 & 5.02308 & 6.33670 & 0.00001 & 0.00004 & 0.00007 & 0.00530 \\
\hline & 4.00000 & 0.75000 & 0.80000 & 0.56588 & 4.05132 & 0.75191 & 0.80795 & 0.00001 & 0.00029 & 0.00002 & 0.00002 \\
\hline & & & 1.80000 & 0.56426 & 4.03625 & 0.75584 & 1.83647 & 0.00001 & 0.00024 & 0.00003 & 0.00018 \\
\hline & & & 6.00000 & 0.56326 & 4.03575 & 0.76524 & 6.34281 & 0.00001 & 0.00029 & 0.00005 & 0.00518 \\
\hline & & 1.70000 & 0.80000 & 0.56371 & 4.04457 & 1.70522 & 0.81023 & 0.00001 & 0.00027 & 0.00003 & 0.00002 \\
\hline & & & 1.80000 & 0.56510 & 4.03096 & 1.70789 & 1.84324 & 0.00001 & 0.00025 & 0.00005 & 0.00018 \\
\hline & & & 6.00000 & 0.56402 & 4.04005 & 1.71104 & 6.32692 & 0.00001 & 0.00028 & 0.00010 & 0.00555 \\
\hline & & 5.00000 & 0.80000 & 0.57054 & 4.04473 & 5.05569 & 0.80854 & 0.00001 & 0.00028 & 0.00011 & 0.00002 \\
\hline & & & 1.80000 & 0.56674 & 4.04515 & 5.03713 & 1.83289 & 0.00001 & 0.00026 & 0.00005 & 0.00019 \\
\hline & & & 6.00000 & 0.56752 & 4.02534 & 5.03339 & 6.41616 & 0.00001 & 0.00030 & 0.00008 & 0.00623 \\
\hline
\end{tabular}


Table 3: Average values of the MLEs and MSEs $(n=750)$ (Continued)

\begin{tabular}{|c|c|c|c|c|c|c|c|c|c|c|c|}
\hline \multicolumn{4}{|c|}{ Parameters } & \multicolumn{4}{|c|}{ MLE } & \multicolumn{4}{|c|}{ MSE } \\
\hline$\alpha$ & $\beta$ & $\lambda$ & $\theta$ & $\widehat{\alpha}$ & $\widehat{\beta}$ & $\widehat{\lambda}$ & $\widehat{\theta}$ & $\widehat{\alpha}$ & $\widehat{\beta}$ & $\widehat{\lambda}$ & $\widehat{\theta}$ \\
\hline \multirow[t]{27}{*}{1.60000} & 0.70000 & 0.75000 & 0.80000 & 1.60478 & 0.70876 & 0.75084 & 0.80801 & 0.00008 & 0.00001 & 0.00002 & 0.00002 \\
\hline & & & 1.80000 & 1.61145 & 0.70597 & 0.75655 & 1.84189 & 0.00008 & 0.00001 & 0.00003 & 0.00019 \\
\hline & & & 6.00000 & 1.61428 & 0.70220 & 0.77321 & 6.44394 & 0.00006 & 0.00001 & 0.00005 & 0.00615 \\
\hline & & 1.70000 & 0.80000 & 1.61395 & 0.70859 & 1.70107 & 0.80946 & 0.00009 & 0.00001 & 0.00004 & 0.00003 \\
\hline & & & 1.80000 & 1.61358 & 0.70582 & 1.70490 & 1.83948 & 0.00007 & 0.00001 & 0.00005 & 0.00018 \\
\hline & & & 6.00000 & 1.61154 & 0.70438 & 1.71673 & 6.36826 & 0.00006 & 0.00001 & 0.00010 & 0.00551 \\
\hline & & 5.00000 & 0.80000 & 1.61761 & 0.70929 & 5.04892 & 0.80768 & 0.00009 & 0.00001 & 0.00006 & 0.00003 \\
\hline & & & 1.80000 & 1.62362 & 0.70546 & 5.03225 & 1.84778 & 0.00007 & 0.00001 & 0.00003 & 0.00020 \\
\hline & & & 6.00000 & 1.61070 & 0.70313 & 5.03132 & 6.41842 & 0.00006 & 0.00001 & 0.00009 & 0.00615 \\
\hline & 1.50000 & 0.75000 & 0.80000 & 1.62054 & 1.51763 & 0.75176 & 0.80878 & 0.00009 & 0.00003 & 0.00002 & 0.00002 \\
\hline & & & 1.80000 & 1.62190 & 1.50938 & 0.75856 & 1.84507 & 0.00007 & 0.00003 & 0.00003 & 0.00019 \\
\hline & & & 6.00000 & 1.61214 & 1.50840 & 0.76819 & 6.39645 & 0.00006 & 0.00003 & 0.00005 & 0.00610 \\
\hline & & 1.70000 & 0.80000 & 1.62118 & 1.52170 & 1.69653 & 0.80479 & 0.00009 & 0.00003 & 0.00003 & 0.00002 \\
\hline & & & 1.80000 & 1.62038 & 1.51451 & 1.70159 & 1.83586 & 0.00007 & 0.00003 & 0.00005 & 0.00020 \\
\hline & & & 6.00000 & 1.61546 & 1.50692 & 1.72025 & 6.40444 & 0.00007 & 0.00004 & 0.00011 & 0.00621 \\
\hline & & 5.00000 & 0.80000 & 1.62582 & 1.51679 & 5.04115 & 0.80779 & 0.00009 & 0.00003 & 0.00006 & 0.00002 \\
\hline & & & 1.80000 & 1.61619 & 1.50696 & 5.03053 & 1.85648 & 0.00007 & 0.00003 & 0.00003 & 0.00020 \\
\hline & & & 6.00000 & 1.61620 & 1.50714 & 5.02909 & 6.42390 & 0.00007 & 0.00004 & 0.00009 & 0.00634 \\
\hline & 4.00000 & 0.75000 & 0.80000 & 1.61269 & 4.05412 & 0.74920 & 0.80642 & 0.00008 & 0.00024 & 0.00002 & 0.00002 \\
\hline & & & 1.80000 & 1.62716 & 4.01927 & 0.76138 & 1.85277 & 0.00007 & 0.00022 & 0.00003 & 0.00020 \\
\hline & & & 6.00000 & 1.61828 & 4.02669 & 0.76628 & 6.37333 & 0.00006 & 0.00025 & 0.00005 & 0.00598 \\
\hline & & 1.70000 & 0.80000 & 1.61558 & 4.03261 & 1.70558 & 0.81251 & 0.00009 & 0.00024 & 0.00004 & 0.00003 \\
\hline & & & 1.80000 & 1.61408 & 4.01968 & 1.71136 & 1.85235 & 0.00007 & 0.00021 & 0.00005 & 0.00019 \\
\hline & & & 6.00000 & 1.61117 & 4.02337 & 1.71928 & 6.40954 & 0.00006 & 0.00026 & 0.00011 & 0.00629 \\
\hline & & 5.00000 & 0.80000 & 1.61807 & 4.03387 & 5.04379 & 0.81147 & 0.00009 & 0.00023 & 0.00007 & 0.00002 \\
\hline & & & 1.80000 & 1.60799 & 4.01091 & 5.02976 & 1.85946 & 0.00007 & 0.00021 & 0.00004 & 0.00019 \\
\hline & & & 6.00000 & 1.61161 & 4.03128 & 5.02220 & 6.35638 & 0.00006 & 0.00025 & 0.00009 & 0.00599 \\
\hline
\end{tabular}

\begin{tabular}{|c|c|c|c|c|c|c|c|c|c|c|c|}
\hline \multirow[t]{27}{*}{3.00000} & \multirow[t]{9}{*}{0.70000} & \multirow[t]{3}{*}{0.75000} & 0.80000 & 3.05264 & 0.70413 & 0.75859 & 0.81615 & 0.00037 & 0.00001 & 0.00002 & 0.00003 \\
\hline & & & 1.80000 & 3.05630 & 0.70331 & 0.76100 & 1.85444 & 0.00030 & 0.00001 & 0.00003 & 0.00021 \\
\hline & & & 6.00000 & 3.03373 & 0.70412 & 0.76736 & 6.39585 & 0.00024 & 0.00001 & 0.00005 & 0.00616 \\
\hline & & \multirow{3}{*}{1.70000} & 0.80000 & 3.04120 & 0.70644 & 1.70278 & 0.81133 & 0.00035 & 0.00001 & 0.00004 & 0.00003 \\
\hline & & & 1.80000 & 3.04692 & 0.70501 & 1.70652 & 1.84705 & 0.00029 & 0.00001 & 0.00005 & 0.00021 \\
\hline & & & 6.00000 & 3.04042 & 0.70654 & 1.70819 & 6.33611 & 0.00024 & 0.00001 & 0.00011 & 0.00617 \\
\hline & & \multirow[t]{3}{*}{5.00000} & 0.80000 & 3.03097 & 0.70531 & 5.03722 & 0.81622 & 0.00036 & 0.00001 & 0.00006 & 0.00003 \\
\hline & & & 1.80000 & 3.04296 & 0.70456 & 5.01822 & 1.84173 & 0.00027 & 0.00001 & 0.00004 & 0.00020 \\
\hline & & & 6.00000 & 3.03330 & 0.70375 & 5.02317 & 6.36912 & 0.00024 & 0.00001 & 0.00009 & 0.00575 \\
\hline & \multirow[t]{9}{*}{1.50000} & \multirow[t]{3}{*}{0.75000} & 0.80000 & 3.04296 & 1.51417 & 0.75276 & 0.80955 & 0.00036 & 0.00003 & 0.00002 & 0.00003 \\
\hline & & & 1.80000 & 3.04633 & 1.50818 & 0.76051 & 1.85468 & 0.00029 & 0.00003 & 0.00003 & 0.00021 \\
\hline & & & 6.00000 & 3.02468 & 1.51543 & 0.76103 & 6.34235 & 0.00023 & 0.00004 & 0.00005 & 0.00619 \\
\hline & & \multirow[t]{3}{*}{1.70000} & 0.80000 & 3.05932 & 1.51239 & 1.70346 & 0.81190 & 0.00038 & 0.00003 & 0.00004 & 0.00003 \\
\hline & & & 1.80000 & 3.03853 & 1.50923 & 1.70824 & 1.85104 & 0.00028 & 0.00003 & 0.00006 & 0.00021 \\
\hline & & & 6.00000 & 3.04623 & 1.50361 & 1.72812 & 6.48593 & 0.00025 & 0.00004 & 0.00011 & 0.00671 \\
\hline & & \multirow[t]{3}{*}{5.00000} & 0.80000 & 3.05534 & 1.51167 & 5.03678 & 0.81451 & 0.00037 & 0.00003 & 0.00005 & 0.00003 \\
\hline & & & 1.80000 & 3.04656 & 1.50660 & 5.02537 & 1.85638 & 0.00028 & 0.00003 & 0.00003 & 0.00021 \\
\hline & & & 6.00000 & 3.02411 & 1.50794 & 5.02220 & 6.40179 & 0.00024 & 0.00003 & 0.00010 & 0.00636 \\
\hline & \multirow[t]{9}{*}{4.00000} & \multirow[t]{3}{*}{0.75000} & 0.80000 & 3.03481 & 4.03586 & 0.75457 & 0.81241 & 0.00034 & 0.00023 & 0.00002 & 0.00003 \\
\hline & & & 1.80000 & 3.03896 & 4.02234 & 0.76138 & 1.86011 & 0.00029 & 0.00021 & 0.00003 & 0.00022 \\
\hline & & & 6.00000 & 3.03312 & 4.00949 & 0.77429 & 6.48198 & 0.00023 & 0.00024 & 0.00005 & 0.00652 \\
\hline & & \multirow[t]{3}{*}{1.70000} & 0.80000 & 3.03135 & 4.03600 & 1.70333 & 0.81134 & 0.00036 & 0.00021 & 0.00004 & 0.00002 \\
\hline & & & 1.80000 & 3.02530 & 4.02071 & 1.71210 & 1.85823 & 0.00028 & 0.00022 & 0.00006 & 0.00021 \\
\hline & & & 6.00000 & 3.03307 & 4.00875 & 1.72817 & 6.49298 & 0.00024 & 0.00024 & 0.00011 & 0.00767 \\
\hline & & \multirow[t]{3}{*}{5.00000} & 0.80000 & 3.06166 & 4.03732 & 5.03859 & 0.81120 & 0.00037 & 0.00022 & 0.00005 & 0.00002 \\
\hline & & & 1.80000 & 3.02559 & 4.03055 & 5.02627 & 1.84456 & 0.00029 & 0.00020 & 0.00004 & 0.00020 \\
\hline & & & 6.00000 & 3.04140 & 4.01970 & 5.02725 & 6.44871 & 0.00024 & 0.00025 & 0.00011 & 0.00697 \\
\hline
\end{tabular}


The Alpha Power Transformation Family: Properties and Applications

Table 4: MLEs (SEs in parentheses), K-S statistics and $p$-values for the first data set.

\begin{tabular}{|c|c|c|c|c|c|c|}
\hline \multirow[t]{2}{*}{ Model } & \multicolumn{4}{|c|}{ Estimates } & \multicolumn{2}{|c|}{ Goodness-of-fit } \\
\hline & $\widehat{\alpha}$ & $\widehat{\beta}$ & $\widehat{\lambda}$ & $\widehat{\theta}$ & $\mathrm{K}-\mathrm{S}$ & $p$-value \\
\hline APEW & $\begin{array}{c}0.59612 \\
(0.61808)\end{array}$ & $\begin{array}{c}1.47480 \\
(0.32760)\end{array}$ & $\begin{array}{c}0.01018 \\
(0.01375)\end{array}$ & $\begin{array}{c}0.51911 \\
(0.16192)\end{array}$ & 0.10282 & 0.43186 \\
\hline MW & $\begin{array}{c}0.04156 \\
(0.09955)\end{array}$ & $\begin{array}{c}0.07005 \\
(0.10295)\end{array}$ & $\begin{array}{c}0.81069 \\
(0.40086)\end{array}$ & & 0.10470 & 0.40896 \\
\hline TW & $\begin{array}{c}0.90117 \\
(0.10920)\end{array}$ & $\begin{array}{c}11.63227 \\
(3.22754)\end{array}$ & $\begin{array}{c}0.00001 \\
(0.38800)\end{array}$ & & 0.10521 & 0.40289 \\
\hline $\mathrm{W}$ & & $\begin{array}{c}11.63219 \\
(1.60170)\end{array}$ & $\begin{array}{c}0.90117 \\
(0.08556)\end{array}$ & & 0.10521 & 0.40288 \\
\hline $\mathrm{APW}$ & $\begin{array}{c}1.13941 \\
(1.26643)\end{array}$ & $\begin{array}{c}0.88948 \\
(0.13198)\end{array}$ & $\begin{array}{c}0.11691 \\
(0.07218)\end{array}$ & & 0.10610 & 0.39239 \\
\hline EW & & $\begin{array}{c}1.38134 \\
(0.38079)\end{array}$ & $\begin{array}{c}0.01613 \\
(0.02404)\end{array}$ & $\begin{array}{c}0.52171 \\
(0.20886)\end{array}$ & 0.10752 & 0.37589 \\
\hline ZBLL & $\begin{array}{c}33.08202 \\
(1.11641)\end{array}$ & $\begin{array}{c}4.10235 \\
(0.87764)\end{array}$ & $\begin{array}{c}0.03794 \\
(0.14918)\end{array}$ & & 0.11081 & 0.33962 \\
\hline APEx & $\begin{array}{c}0.58639 \\
(0.46447)\end{array}$ & & $\begin{array}{c}0.07192 \\
(0.01747)\end{array}$ & & 0.12293 & 0.22668 \\
\hline BW & $\begin{array}{c}9.72347 \\
(19.48573)\end{array}$ & $\begin{array}{c}0.09977 \\
(0.04361)\end{array}$ & $\begin{array}{c}62.50587 \\
(52.79583)\end{array}$ & $\begin{array}{c}18.06019 \\
(18.75014)\end{array}$ & 0.13302 & 0.15643 \\
\hline
\end{tabular}

at the National Physical Laboratory, England. These data were used by Afify et al. (2016) to fit the Weibull Fréchet distribution.

We compare the fits of the new distribution and its sub-models APEx and W distributions for the first data set, and APW, APEx and W distributions for the second data set. Further, we consider some other competitive distributions, namely: EW (Mudholkar and Srivastava, 1993), transmuted Weibull (TW) (Aryall and Tsokos, 2011), modified Weibull (MW) (Sarhan and Zaindin, 2009), beta Weibull (BW) (Lee et al., 2007), and Zografos-Balakrishnan log-logistic (ZBLL) (Zografos and Balakrishnan, 2009) models.

Tables 4 and 5 give the MLEs and the corresponding standard errors (SEs) in parentheses of the parameters for all fitted models and the Kolmogorov-Smirnov (K-S) statistics and p-values for the first and the second data sets, respectively. Since the APEW distribution has the lowest $\mathrm{K}-\mathrm{S}$ values and largest $p$-values among all fitted models, it could be chosen as the best model for both data sets. The histograms and fitted pdfs and cdfs for the first and second data are displayed in Figure 3 and Figure 4, respectively. 
Table 5: MLEs (SEs in parentheses), K-S statistics and $p$-values for the second data set.

\begin{tabular}{|c|c|c|c|c|c|c|c|}
\hline \multirow[t]{2}{*}{ Model } & \multicolumn{5}{|c|}{ Estimates } & \multicolumn{2}{|c|}{ Goodness-of-fit } \\
\hline & $\widehat{\alpha}$ & $\widehat{\beta}$ & $\widehat{\lambda}$ & $\widehat{\theta}$ & $\widehat{b}$ & K-S & $p$-value \\
\hline APEW & $\begin{array}{c}15.08459 \\
(22.32652)\end{array}$ & $\begin{array}{c}5.51065 \\
(1.38647)\end{array}$ & $\begin{array}{c}0.09021 \\
(0.09190)\end{array}$ & $\begin{array}{c}0.58711 \\
(0.33502)\end{array}$ & & 0.11590 & 0.36592 \\
\hline APW & $\begin{array}{c}10.85645 \\
(12.71804)\end{array}$ & $\begin{array}{c}4.48359 \\
(0.76269)\end{array}$ & $\begin{array}{c}0.19478 \\
(0.10824)\end{array}$ & & & 0.12249 & 0.30099 \\
\hline ZBLL & $\begin{array}{c}1.82436 \\
(0.04660)\end{array}$ & $\begin{array}{c}18.41059 \\
(3.06176)\end{array}$ & $\begin{array}{c}0.25140 \\
(0.06169)\end{array}$ & & & 0.13054 & 0.23325 \\
\hline MW & $\begin{array}{c}0.03106 \\
(0.04347)\end{array}$ & $\begin{array}{c}0.04071 \\
(0.02464)\end{array}$ & $\begin{array}{c}6.38068 \\
(0.96423)\end{array}$ & & & 0.13311 & 0.21426 \\
\hline $\mathrm{EW}$ & & $\begin{array}{c}7.28412 \\
(1.48698)\end{array}$ & $\begin{array}{c}0.01941 \\
(0.02109)\end{array}$ & $\begin{array}{c}0.67128 \\
(0.22097)\end{array}$ & & 0.14624 & 0.13508 \\
\hline TW & $\begin{array}{c}5.97479 \\
(0.74495)\end{array}$ & $\begin{array}{c}1.80961 \\
(0.07554)\end{array}$ & $\begin{array}{c}0.92496 \\
(0.21932)\end{array}$ & & & 0.15191 & 0.10918 \\
\hline W & & $\begin{array}{c}1.62811 \\
(0.03709)\end{array}$ & $\begin{array}{c}5.78070 \\
(0.57610)\end{array}$ & & & 0.15224 & 0.10784 \\
\hline APEx & $\begin{array}{l}4787253.00000 \\
(23726.62000)\end{array}$ & & $\begin{array}{c}2.10993 \\
(0.09894)\end{array}$ & & & 0.22224 & 0.00397 \\
\hline BW & $\begin{array}{c}0.91988 \\
(0.14720)\end{array}$ & $\begin{array}{c}7.01279 \\
(0.88915)\end{array}$ & $\begin{array}{c}0.44933 \\
(0.18083)\end{array}$ & $\begin{array}{c}0.04960 \\
(0.04595)\end{array}$ & & 0.36508 & $1 e-7$ \\
\hline
\end{tabular}


The Alpha Power Transformation Family: Properties and Applications
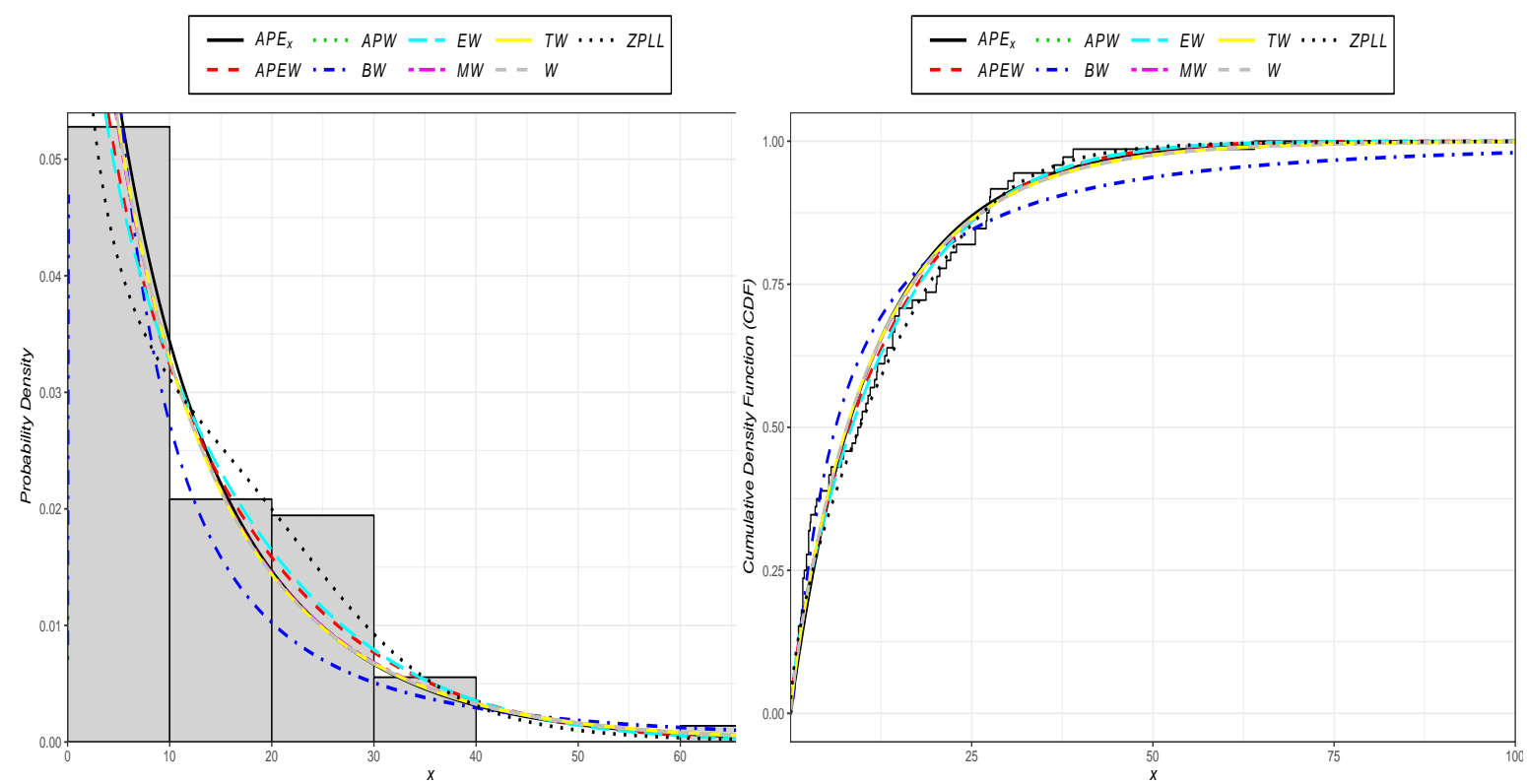

Figure 3: The fitted pdfs and cdfs of the APEW model and other fitted models for the first data set.
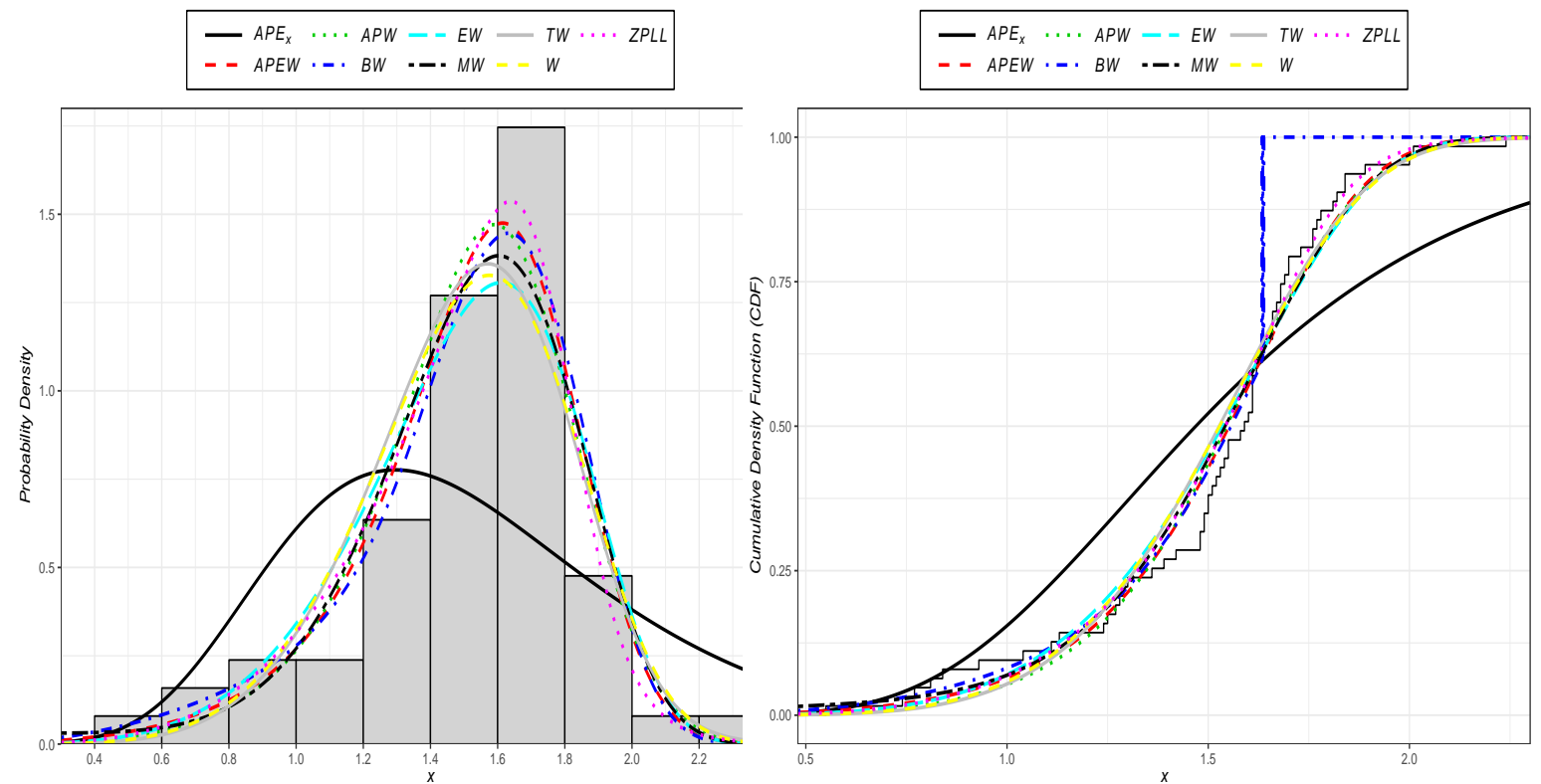

Figure 4: The fitted pdfs and cdfs of the APEW model and other fitted models for the second data set.

\section{Concluding remarks}

In this paper, we investigate some mathematical properties of the alpha power transformation family (Mahdavi and Kundu, 2017) and propose a new four-parameter model, named the alpha power exponentiated Weibull (APEW) distribution, which extends the well-known exponentiated Weibull distribution pioneered by Mudholkar et al. (1995). The APEW model is motivated by the wide use of the Weibull distribution in reliability theory and also for the fact that the generalization provides 
more flexibility to analyze positive real data. We derive explicit expressions for some of its mathematical quantities. We discuss the maximum likelihood estimation of the model parameters and provide some simulation results to assess the performance of the proposed distribution. Two applications to real data illustrate that the new distribution provides consistently better fits than other nested and non-nested models. We hope that the new model will attract wider applications in areas such as engineering, survival and lifetime data, hydrology, economics (income inequality) and others.

\section{References}

1. Afify, A., Yousof, H., Cordeiro, G., Ortega, E., and Nofal, Z. (2016). The Weibull Fréchet distribution and its applications. Journal of Applied Statistics, 43:2608-2626.

2. Aryall, G. and Tsokos, C. (2011). Transmuted Weibull distribution: A generalization of the Weibull probability distribution. European Journal of Pure and Applied Mathematics, 2:89-102.

3. Choulakian, V. and Stephens, M. (2001). Goodness-of-Fit tests for the generalized Pareto distribution. Technometrics, 43:478-484.

4. Lee, C., Famoye, F., and Olumolade, O. (2007). Weibull distribution: some properties and applications to censored data. Journal of Modern Applied Statistical Methods, 6:173-186.

5. Mahdavi, A. and Kundu, D. (2017). A new method for generating distributions with an application to exponential distribution. Communications in Statistics - Theory and Methods, 46:6543-6557.

6. Mudholkar, G. and Srivastava, D. (1993). Exponentiated Weibull family for analyzing bathtub failure-real data. IEEE Transactions on Reliability, 42:299-302.

7. Mudholkar, G., Srivastava, D., and Friemer, M. (1995). The exponentiated Weibull family: A reanalysis of the bus-motor-failure data. Technometrics, $37: 436-445$.

8. Mudholkar, G., Srivastava, D., and Kollia, G. (1996). A generalization of the Weibull distribution with application to the analysis of survival data. Journal of the American Statistical Association, 91:1575-1583.

9. Nadarajah, S., Cancho, V., and Ortega, M. (2013). The geometric exponential Poisson distribution. Statistical Methods and Applications, 22:355380 .

10. Nassar, M., Alzaatreh, A., Mead, M., and Abo-Kasem, O. (2017). Alpha power Weibull distribution: Properties and applications. Communications in Statistics - Theory and Methods, 46(20):10236-10252. 
The Alpha Power Transformation Family: Properties and Applications

11. R Core Team (2019). R: A Language and Environment for Statistical Computing. R Foundation for Statistical Computing, Vienna, Austria.

12. Rayleigh, F. (1880). Xii. on the resultant of a large number of vibrations of the same pitch and of arbitrary phase. The London, Edinburgh, and Dublin Philosophical Magazine and Journal of Science, 10(60):73-78.

13. Sarhan, A. and Zaindin, M. (2009). Modified Weibull distribution. Applied Sciences, 11:123-136.

14. Smith, R. and Naylor, J. (1987). A comparison of maximum likelihood and Bayesian estimators for the three-parameter Weibull distribution. Applied Statistics, 36:358-369.

15. Weibull, W. (1951). A statistical distribution function of wide applicability. Journal of Applied Mechanics, 18:293-297.

16. Zografos, K. and Balakrishnan, N. (2009). On families of beta-and generalized gamma-generated distributions and associated inference. Statistical Methodology, 6:344-362. 